\title{
Accuracy of a semiquantitative method for Dermal Exposure Assessment (DREAM)
}

\author{
B van Wendel de Joode, R Vermeulen, J J van Hemmen, W Fransman, H Kromhout
}

Occup Environ Med 2005;62:623-632. doi: 10.1136/oem.2004.018564

See end of article for authors' affiliations

Correspondence to: Dr H Kromhout,

Environmental and Occupational Health

Division, Institute for Risk

Assessment Sciences,

Utrecht University, PÓ Box

80176,3508 TD Utrecht,

Netherlands; h.kromhout@ iras.uu.nl

Accepted 18 March 2005
Background: The authors recently developed a Dermal Exposure Assessment Method (DREAM), an observational semiquantitative method to assess dermal exposures by systematically evaluating exposure determinants using pre-assigned default values.

Aim: To explore the accuracy of the DREAM method by comparing its estimates with quantitative dermal exposure measurements in several occupational settings.

Methods: Occupational hygienists observed workers performing a certain task, whose exposure to chemical agents on skin or clothing was measured quantitatively simultaneously, and filled in the DREAM questionnaire. DREAM estimates were compared with measurement data by estimating Spearman correlation coefficients for each task and for individual observations. In addition, mixed linear regression models were used to study the effect of DREAM estimates on the variability in measured exposures between tasks, between workers, and from day to day.

Results: For skin exposures, spearman correlation coefficients for individual observations ranged from 0.19 to 0.82 . DREAM estimates for exposure levels on hands and forearms showed a fixed effect between and within surveys, explaining mainly between-task variance. In general, exposure levels on clothing layer were only predicted in a meaningful way by detailed DREAM estimates, which comprised detailed information on the concentration of the agent in the formulation to which exposure occurred.

Conclusions: The authors expect that the DREAM method can be successfully applied for semiquantitative dermal exposure assessment in epidemiological and occupational hygiene surveys of groups of workers with considerable contrast in dermal exposure levels (variability between groups $>1.0$ ). For surveys with less contrasting exposure levels, quantitative dermal exposure measurements are preferable. l: n occupational epidemiology there has been an increasing trend to use experts, such as occupational hygienists, to semiquantitatively assess exposure to chemical agents, because measuring exposure levels is often considered too expensive, time consuming, or, in the case of retrospective surveys, impossible. Several authors have evaluated the accuracy of semiquantitative exposure assessment methods for occupational exposures to chemical agents..$^{2-10}$ All except the studies on exposure to pesticides by de Cock et al, ${ }^{4}$ and exposure to paint by Brouwer et al ${ }^{7}$ exclusively focused on airborne exposure levels. Validated semiquantitative occupational dermal exposure assessment methods applicable at workplaces for a broad range of substances are practically non-existent.

Ignoring the dermal route for exposure assessment in epidemiological studies potentially results in inaccurate and imprecise exposure estimates, which may lead to a loss of power, precision, and attenuation in health risks estimates. ${ }^{11-13}$ Proper assignment of dermal exposure levels in epidemiological surveys requires knowledge about level of exposure (for example, intensity, exposed surface areas, duration), exposure variability (between tasks, workers, and body location), and variability in uptake..$^{13}$

We recently developed a Dermal Exposure Assessment Method (DREAM), ${ }^{14}$ an observational semiquantitative method to assess dermal exposures by systematically evaluating exposure determinants using pre-assigned default values based on a conceptual model for dermal exposure proposed by Schneider et al. ${ }^{15}$ The method is generic and designed for dermal exposure assessment in epidemiological and occupational hygiene surveys. The outcome is a numerical estimate for the dermal exposure level encountered by workers performing a certain task or job. In a previous study on the reliability of DREAM, we showed good interobserver agreement with intraclass correlation coefficients ranging from 0.68 to 0.87 for total dermal exposure estimates. ${ }^{16}$ Also, we successfully applied DREAM for grouping of dermal exposure levels to metal working fluids (MWF) among machine operators for an epidemiological study on dermatitis. ${ }^{17}$ The aim of the present study was to explore the accuracy of the DREAM method by comparing its estimates with quantitative dermal exposure measurements in several occupational settings.

\section{MATERIAL AND METHODS}

\section{Dermal exposure assessment method}

The basis for DREAM is the conceptual model for dermal exposure of Schneider and colleagues, ${ }^{15}$ who described mechanisms by which dermal exposure can occur. The most important exposure routes distinguished in this model are emission, transfer, and deposition, resulting in exposure on outer clothing and skin. Schneider et al ${ }^{15}$ defined emission as dermal exposure occurring directly from the source of exposure, transfer as exposure due to contact with contaminated surfaces, and deposition as exposure through skin contact with small, airborne particles.

DREAM consists of a multiple choice questionnaire (inventory part of DREAM) that the user fills in while observing a worker performing a certain task. Included are questions about: (1) the probability and intensity of the main

Abbreviations: $B Z$, benzene; $C P$, cyclophosphamide; $D E G B E$, di-ethylglycol-butyl-ether; DREAM, Dermal Exposure Assessment Method; DU, DREAM units; GM, geometric mean; GSD, geometric standard deviation; LOD, limit of detection; MWF, metal working fluids; OS, organic solvents; $\mathrm{TL}$, tolvene 
dermal exposure routes: emission, deposition, and transfer for nine body parts; (2) information on clothing layer (kind of material covering skin, replacement frequency of clothing, percentage of time gloves are being worn) for the worker performing the task; (3) physical and chemical characteristics of the substance to which dermal exposure occurs; and (4) percentage of working time a task is performed.

Each answer of the questionnaire matches with an a priori assigned value. For example, the question on probability of emission on hands is defined as "(covered) hands are exposed by direct release of agent from a source, or by immersion?" with answers (and assigned values in parenthesis) "no unlikely $(0)$; yes occasionally, $<10 \%$ of task duration ( 1 ); yes repeatedly, $10-50 \%$ of task duration (3); yes almost constantly, $\geqslant 50 \%$ of task duration (10). The default values increase and decrease on a log scale-that is, $0.3,1,3,10$-as used by Cherrie and colleagues (1996) in their model for evaluating airborne exposures. The directions of the default values of DREAM (increasing $v$ decreasing exposure) are derived from literature and expert judgment.

In the evaluation part of DREAM, the assigned values are used in an algorithm resulting in numerical estimates for exposure levels on both outside clothing layer (potential dermal exposure) and, after taking into account the protective effect of clothing, on skin (actual dermal exposure). Estimates are provided for nine individual body parts (hands, forearms, upper arms, head, torso front, back, lower abdomen to knees, lower legs, and feet) as well as for total body exposure.

DREAM assesses dermal exposure levels relatively to task duration. In order to take into account the time a task is performed, and thus exposure time, the DREAM estimate is multiplied by its task duration and divided by total working time assessed on the same timescale, resulting in the total dermal exposure level for each body part, and total body exposure, during a working day expressed in DREAM units (DU). In case different tasks are performed on a working day, the DREAM estimates of the tasks are summed in order to estimate total dermal exposure levels. For a detailed description of the methodology of the DREAM method we refer to van Wendel de Joode et al. ${ }^{14}$

\section{Study design}

In six exposure surveys, occupational hygienists filled in the DREAM questionnaire while observing a worker performing one of the tasks described in table 1 during 5-30 minutes. Dermal exposure was measured quantitatively at the same time for the full task, or shift, duration. Training for occupational hygienists included (1) a short explanation of the conceptual model of Schneider and colleagues (1999) and the DREAM questionnaire, (2) agreement among the observers on where tasks started and ended, and (3) one to five practice runs to familiarise observers with the application of the method.

\section{Description of performed surveys}

In surveys 1, 2, 5, and 6, one occupational hygienist in each survey performed observations, while in surveys 3 and 4 two occupational hygienists performed observations. For surveys 1 to 4 the DREAM questionnaire was filled in for each individual worker. For surveys 5 and 6 the DREAM questionnaire was filled in at task level. Table 1 describes the six exposure surveys. Details of the methodology of five out of the six surveys have been published elsewhere. ${ }^{17-21}$ Dermal exposure was assessed to metal working fluids (MWF) (survey l, manufacturing of motor blocks), organic solvents (OS) (survey 2, spray painting), cyclophosphamide (CP) (survey 3, handling of antineoplastic drugs in hospitals), di-ethyl-glycol-butyl-ether (DEGBE) (survey 4, chemical industry), benzene (BZ) (survey 5, petrochemical industry), and toluene (TL) (survey 6, shoe manufacturing) for workers performing different tasks. Measurements were performed during the time a worker performed a specific task (surveys 2, 3, and 4) or during (part of) a shift (surveys 1, 2, 5, and 6). For shift based measurements, the measurement time generally also coincided with task duration, because tasks were defined less specifically in these situations. Measurements were conducted on skin and clothing layer using surrogate skin techniques (pads, gloves), ${ }^{22}$ removal techniques (washing, wiping), ${ }^{24}$ and fluorescent tracer techniques (Video Imaging Technique for Assessing Exposure (VITAE))..$^{24-27}$

\section{The comparison of DREAM estimates with quantitative data}

DREAM estimates (expressed in DU) were compared with measured exposure levels on skin and clothing expressed as $\mu \mathrm{g} / \mathrm{cm}^{2}$. For shift based measurements, if different tasks were performed during a shift, time weighted DREAM estimates (DU) were summed in order to obtain DREAM estimates at shift level.

Comparisons were made for hands, forearms (only for survey 1), and legs, as measurement data with more than $50 \%$ of samples above the limit of detection (LOD) were available for these body parts (see table 2). In case of exposure to benzene, we assumed that skin exposure levels measured on wrists represented skin exposure levels of hands in order to be able to compare these data with the other surveys. Note that for exposure to organic solvents (survey 2) on clothing layer only measurements were available for four (spray painting tasks) out of the six tasks for which skin exposure was measured (table 1).

As mentioned before, DREAM takes into account the concentration of active ingredient (AI) in the substance to which exposure occurs. In the evaluation model of DREAM, the concentration of AI is a categorical estimate with value 1 (concentration of AI of interest $>90 \%$ ), 0.3 (concentration of AI of interest $1-90 \%$ ), or 0.1 (concentration of AI of interest $<1 \%)$. In addition to the original DREAM estimates, we calculated detailed DREAM estimates $\left(\mathrm{DU}_{\mathrm{DET}}\right)$ using the actual concentration of active ingredient of interest in the substance for which exposure was assessed, expressed as \% weight-weight, to allow for a more specific estimate of dermal exposure. For survey l (exposure to MWF), the detailed DREAM estimates were equal to the original DREAM estimates as the concentration of active ingredient of interest (MWF) was $100 \%$. The limit of detection (LOD) of DREAM estimates was defined as the lowest DREAM estimate greater than zero DU. LODs on skin and clothing layer were 0.008 and $0.03 \mathrm{DU}$, respectively.

\section{Statistical analyses}

Statistical analyses were performed in SAS version 8.2 (SAS Institute, Cary, NC, USA). Non-detectable dermal exposure levels and DREAM estimates were set to $1 / \sqrt{2}$ of the LOD. Both measured dermal exposure levels and DREAM estimates followed a lognormal distribution. In order to study whether the DREAM method is able to rank dermal exposure levels between surveys, DREAM estimates were compared with measurement data by estimating Spearman rank correlation coefficients for each task within a company, and for individual observations.

For surveys with observations for individual workers (surveys 1 to 4 ), mixed linear regression models were used to estimate variance components of log-transformed measured exposure levels for tasks, workers, and day to day (model A). Subsequently, the effect of the log-transformed DREAM estimates on the log-transformed measured 


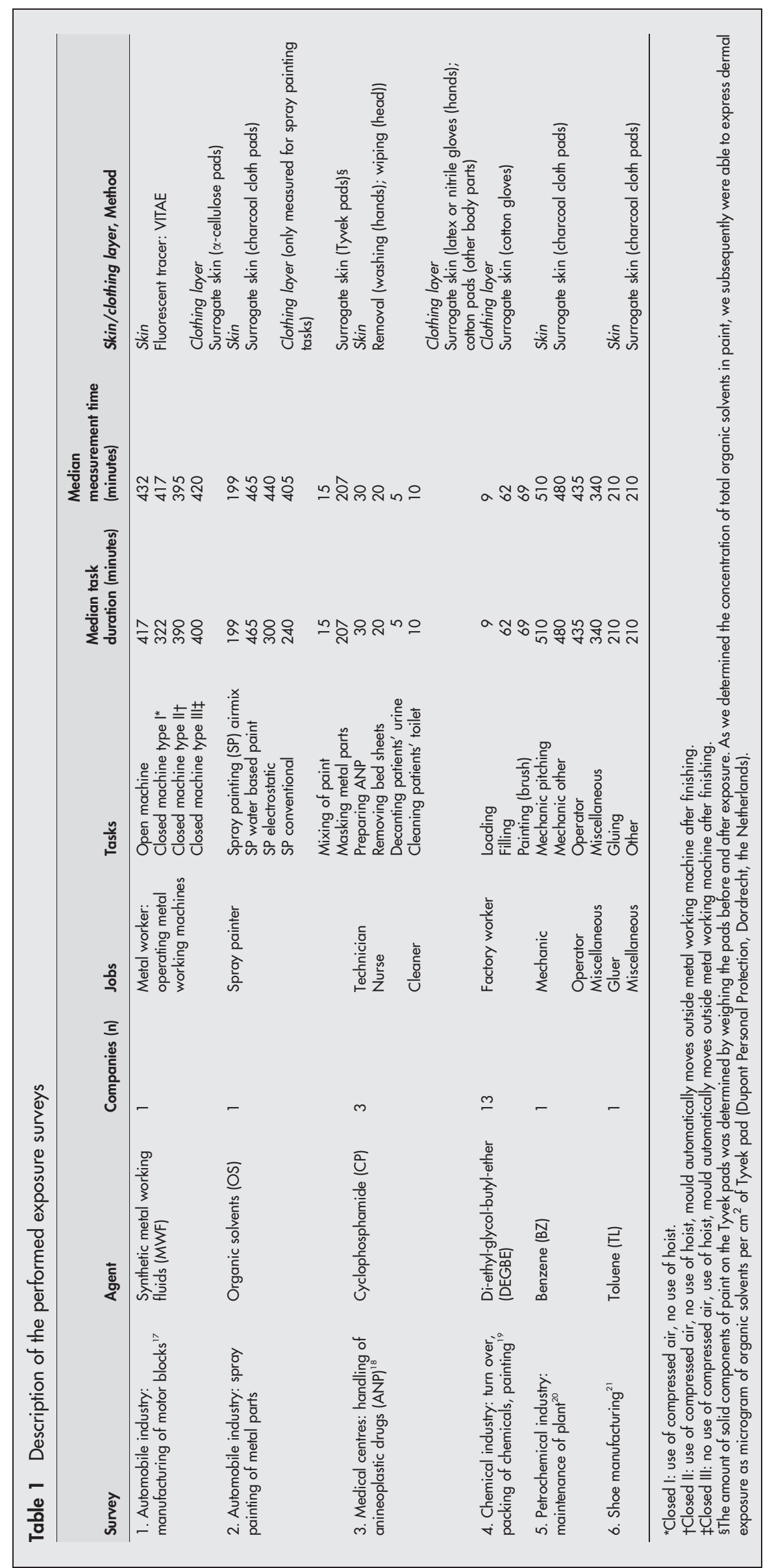


Table 2 Description of collected data with geometric means (GMs) and geometric standard deviations (GSDs) of measurements $\left(\mathrm{\mu g} / \mathrm{cm}^{2}\right)$ and DREAM estimates (DU)

\begin{tabular}{|c|c|c|c|c|c|c|c|}
\hline Study/agent & Method & Body part & $\mathbf{n}^{*}$ & $\mathrm{~K} \dagger$ & $\%>$ LOD & $\begin{array}{l}\text { GM (GSD) } \\
\text { measurements } \\
\left(\mu \mathrm{g} / \mathrm{cm}^{2}\right)\end{array}$ & DREAM (DU) \\
\hline \multicolumn{8}{|l|}{ Skin layer } \\
\hline \multirow[t]{3}{*}{ 1. MWF } & \multirow[t]{3}{*}{ VITAE } & Hands & 41 & 32 & 85 & $626(6.2)$ & $10(2.9)$ \\
\hline & & Forearms & 41 & 32 & 88 & $576(5.4)$ & $4(5.2)$ \\
\hline & & Head & 41 & 32 & 32 & $248(2.6)$ & $5.10^{-1}(43.1)$ \\
\hline \multirow[t]{2}{*}{ 2. OS } & \multirow[t]{2}{*}{ Surrogate skin } & Hand (dominant) & 16 & 10 & 88 & $291(5.8)$ & $6.10^{-1}(9.9)$ \\
\hline & & Upper leg (right) & 27 & 16 & 93 & $525(3.9)$ & $4.10^{-1}(17.6)$ \\
\hline \multirow[t]{2}{*}{ 3. $C P$} & \multirow[t]{2}{*}{ Removal } & Hands & 42 & 28 & 38 & $3.10^{-5}(3.5)$ & $2.10^{-3}(3.4)$ \\
\hline & & Head & 41 & 27 & 24 & $7.10^{-4}(1.9)$ & $2.10^{-4}(2.5)$ \\
\hline 5. $B Z$ & Surrogate skin & Wrist (dominant) & 42 & 37 & 64 & $2.10^{-1}(17.7)$ & $1.10^{-1}(9.1)$ \\
\hline 6. $\mathrm{TL}$ & Surrogate skin & Hand (dominant) & 127 & 74 & 83 & $14(20.7)$ & $3.10^{-2}(75.5)$ \\
\hline \multicolumn{8}{|l|}{ Clothing layer } \\
\hline \multirow[t]{4}{*}{ 1. MWF } & \multirow[t]{4}{*}{ Surrogate skin } & Upper arm & 58 & 45 & 22 & $1106(2.0)$ & $5.10^{-2}(11.5)$ \\
\hline & & Torso front & 58 & 45 & 7 & $936(1.7)$ & $3.10^{-1}(28.7)$ \\
\hline & & Torso back & 9 & 8 & 11 & $1033(2.3)$ & $1.10^{-2}(2.3)$ \\
\hline & & Upper legs & 56 & 44 & 71 & 11024 (3.0) & $5(9.3)$ \\
\hline \multirow[t]{2}{*}{ 2. OS } & \multirow{9}{*}{$\begin{array}{l}\text { Surrogate skin } \\
\text { (weighing) } \\
\text { Surrogate skin }\end{array}$} & Hands & 61 & 33 & 100 & $1469(2.8)$ & $17(1.4)$ \\
\hline & & Upper leg (left) & 62 & 33 & 100 & $475(3.4)$ & $19(1.4)$ \\
\hline \multirow[t]{7}{*}{ 3. $\mathrm{CP}$} & & Hands & 42 & 28 & 45 & $4.10^{-5}(8.9)$ & $3.10^{-2}(5.3)$ \\
\hline & & Fore arms & 42 & 28 & 5 & $5.10^{-5}(3.3)$ & $6.10^{-3}(5.5)$ \\
\hline & & Upper arms & 12 & 9 & 17 & $5.10^{-5}(1.8)$ & $2.10^{-3}(3.6)$ \\
\hline & & Torso front & 13 & 10 & 8 & $9.10^{-5}(1.8)$ & $5.10^{-3}(10.7)$ \\
\hline & & Torso back & 13 & 10 & 15 & $9.10^{-5}(1.4)$ & $1.10^{-3}(3.2)$ \\
\hline & & Upper legs & 11 & 8 & 0 & $4.10^{-5}(1.0)$ & $1.10^{-3}(3.7)$ \\
\hline & & Lower legs & 13 & 10 & 0 & $7.10^{-5}(1.0)$ & $1.10^{-3}(3.2)$ \\
\hline 4. DEGBE & Surrogate skin & Hands & 50 & 36 & 100 & $31(37.3)$ & $4.10^{-1}(4.4)$ \\
\hline
\end{tabular}

exposure levels and the estimated variance components was estimated by adding the variable "DREAM estimate" as a fixed effect to model A (model B). The effect of the detailed DREAM estimate on the measured exposure levels and the estimated variance components was estimated by adding the variable "detailed DREAM estimate" as a fixed effect to model A (model C).

$$
\begin{aligned}
& Y_{h i}=\operatorname{Ln}\left(X_{\text {hij }}\right)=\left[\gamma_{00}\right]+\left[v_{0 j}+v_{1 i}+\epsilon_{\text {hij }}\right](\text { model A) } \\
& \mathrm{Y}_{\mathrm{hij}}=\operatorname{Ln}\left(X_{\mathrm{hij}}\right)=\left[\gamma_{00}+\gamma_{01} \cdot \operatorname{LN}\left(\text { DREAM }_{\mathrm{hij}}\right)\right]+\left[v_{0 \mathrm{j}}+v_{1 \mathrm{i}}+\epsilon_{\mathrm{hij}}\right] \\
& \text { (model B) } \\
& \mathrm{Y}_{\mathrm{hij}}=\operatorname{Ln}\left(\mathrm{X}_{\mathrm{hij}}\right)=\left[\gamma_{00}+\gamma_{01} \cdot \operatorname{LN}\left(\text { DREAM }_{\text {DEThij }}\right)\right]+\left[v_{0 \mathrm{j}}+v_{\mathrm{li}}+\epsilon_{\mathrm{hij}}\right] \\
& \text { (model C) }
\end{aligned}
$$

$Y_{h i j}=$ the natural logarithm of the exposure concentration $\left(X_{h i j}\right)$ measured for the $j$ th task of the $i$ th worker on the $h$ th day; $\gamma_{00}=$ the true underlying mean of log-transformed exposure averaged over all groups; $\gamma_{01}=$ fixed effect of logged DREAM estimates measured for the $j$ th task of the $i$ th subject on the $h$ th day; $v_{0 j}=$ random effect of the $j$ th task; $v_{l i}=$ random effect of the $i$ th worker; $\epsilon_{i j}=$ random error (within-worker; day to day variation).

\section{RESULTS}

Table 2 summarises collected measurement data and DREAM estimates for each survey. Figure 1 shows DREAM estimates (DU) plotted against measured exposure levels $\left(\mu \mathrm{g} / \mathrm{cm}^{2}\right)$ on skin and clothing layer of hands and legs, both on log scale. From table 2 as well as figure 1 , it can be seen that variability in measured exposure levels and DREAM estimates was extremely large between surveys. Geometric mean (GM) levels of measured skin exposures varied by $8-10$ orders of magnitude between surveys, and geometric means of DREAM estimates varied by 5-6 orders of magnitude. Within studies, geometric mean levels of measured exposure and DREAM estimates generally varied by less than $1-2$ orders of magnitude. For exposure on clothing layer, GMs of measured exposure levels varied by 9-10 orders of magnitude between surveys, whereas DREAM estimates varied slightly less by 4-6 orders of magnitude (fig 1).

Between surveys, for skin exposure on hands, DREAM estimates correlated well with measured exposure levels, for task based as well as individual observations (Spearman rank correlation coefficients $r=0.78$ and $r=0.82$, respectively) (fig 1). Within surveys, skin exposures on hands at task level could be ranked statistically significant by the DREAM method for two out of four surveys $(r=0.94$ and $r=1.0)$, and for individual observations for two out of three surveys $(r=0.44$ and $r=0.79)$. For skin exposure on other body parts, DREAM moderately ranked exposures for individual observations on forearms (exposure to MWF, $r=0.47$, $\mathrm{p}<0.01(\mathrm{n}=41))$, and did not statistically significant rank exposures on upper legs (exposure to OS, $r=0.30(\mathrm{n}=27)$, $\mathrm{p}=0.12)$ (data not presented).

Between surveys, for exposure on outer clothing layer, DREAM estimates correlated with measured exposure levels on gloves, although less well than for the data of the skin layer (Spearman's $r=0.56$ for task based and $r=0.72$ for individual observations) (fig 1). For clothing layer of legs, DREAM estimates did not significantly correlate with measured exposure levels. Within surveys, exposures could only be ranked in a meaningful way for exposure to MWF on legs $(r=0.53)$.

In figure 2 DREAM estimates and detailed DREAM estimates (DREAM $\mathrm{DET}_{\mathrm{DET}}$ ) are plotted against measurement data $\left(\mu \mathrm{g} / \mathrm{cm}^{2}\right)$, including only data for which the detailed DREAM estimates could be calculated. Between surveys, detailed DREAM estimates performed similar to original DREAM estimates for both exposure on skin and clothing. Within surveys, the detailed DREAM estimates performed slightly worse for skin exposure of hands (fig 2), and performed better for skin exposure on legs $\left(\right.$ DREAM $_{\mathrm{DET}}$ : $r=0.44(\mathrm{p}=0.02) \quad v$ DREAM $_{\mathrm{ORG}}: r=0.30(\mathrm{p}=0.12) \quad($ data not shown)). Within surveys, for exposure on clothing layer 

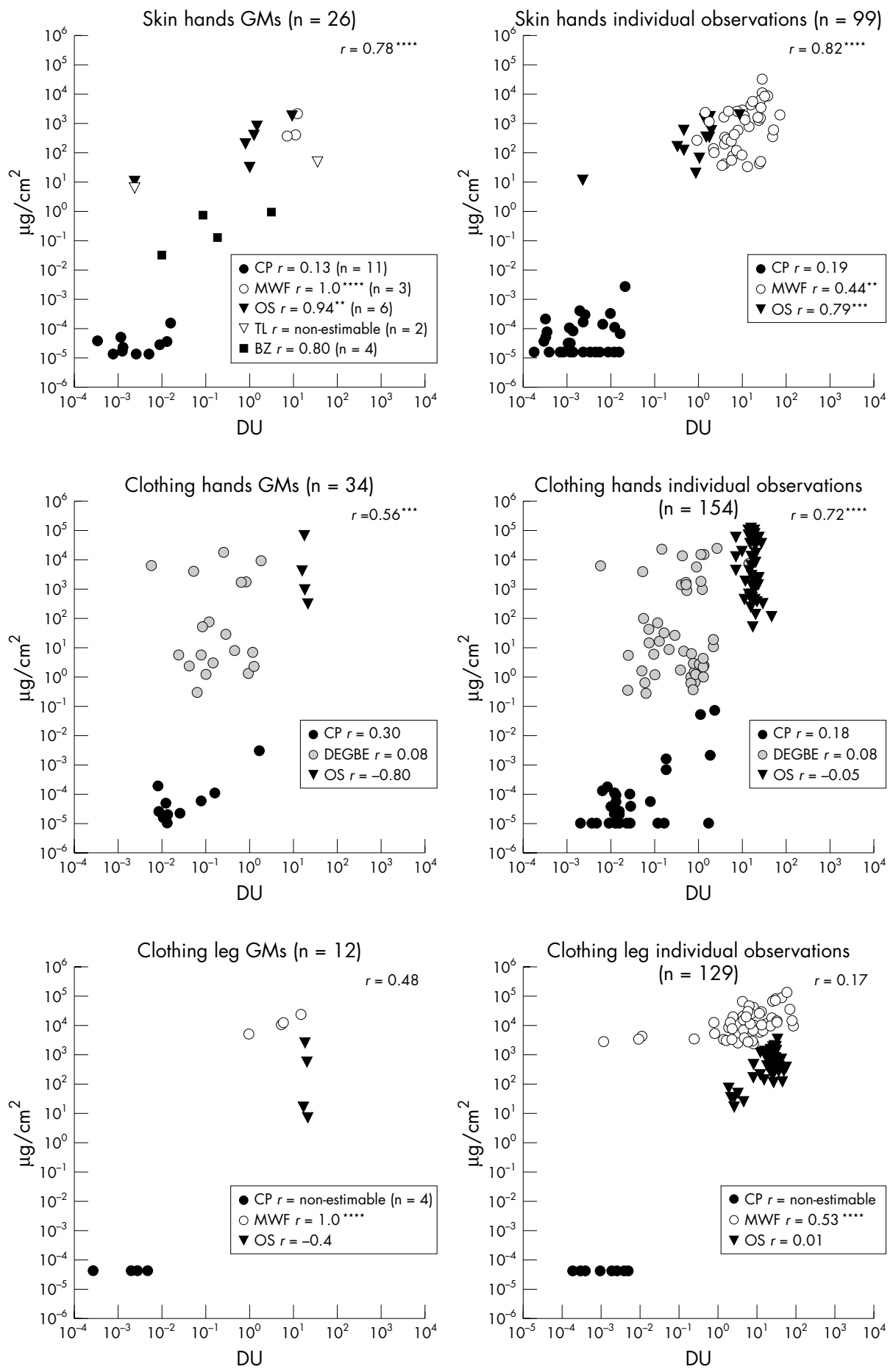

Figure 1 DREAM estimates (DU) plotted against measured exposure levels $\left(\mu \mathrm{g} / \mathrm{cm}^{2}\right)$ on skin and clothing layer of hands and legs, with Spearman rank correlation coefficients $(r)$. Note that for individual observations for skin exposure on hands no data of survey 5 (benzene) and 6 (tolvene) could be included, as only task based observations were performed. However, excluding benzene and tolvene from correlations at task level did not change the found relation (Spearman's $r=0.78 \mathrm{p}<0.0001, \mathrm{n}=20$ ). Note that for exposure to organic solvents on clothing layer only measurements were available for four out of the six tasks for which skin exposure was measured.

of hands, the detailed DREAM estimates also performed better than the original DREAM estimates $(r=0.51 v 0.44(\mathrm{CP})$, $r=0.55 v 0.08$ (DEGBE), $r=0.38 v-0.05$ (OS)) whereas for clothing layer of legs results were similar (fig 2).

In table 3 and 4 results of the mixed linear regression models are presented for body parts with more than $50 \%$ of samples above LOD. For skin exposures, DREAM estimates showed a significant fixed effect between and within surveys for all body parts except for exposure on legs (spray painting), explaining $11 \%-68 \%$ of total variance. In general, the DREAM estimates explained mainly variability in measured exposure levels between tasks, to a lesser extent between workers performing the same task, and not between days. For exposures on clothing layer, a fixed effect of DREAM estimates on the measured exposure levels was present for both hands and legs, between as well as within 

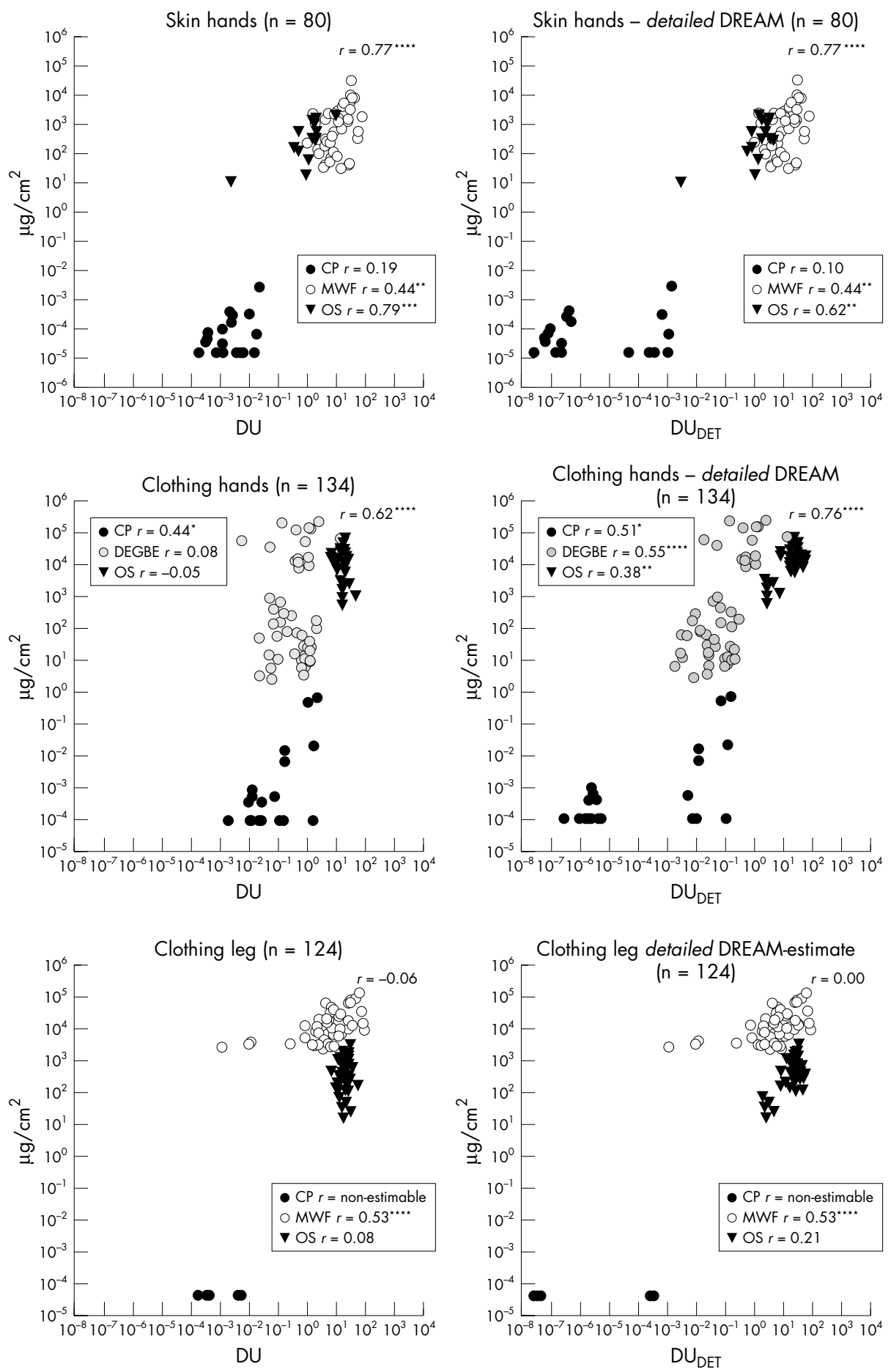

Figure 2 DREAM estimates (DU) and detailed DREAM estimates (DU $\left.U_{D E T}\right)$ plotted against measured exposure levels $\left(\mu \mathrm{g} / \mathrm{cm}^{2}\right)$ on skin and clothing layer of hands and legs with Spearman rank correlation coefficients $(r)$, including only data for which the actual concentration of the active ingredient of interest in the substance to which exposure was assessed, was known.

surveys, except for exposure to organic solvents (spray painting). Again, DREAM estimates explained between-task variance (hands and legs) and between-worker variance (hands) and no day to day variance. Detailed DREAM estimates explained a larger amount of total variance (up to $76 \%$ ) than original DREAM estimates (up to $48 \%$ ). The detailed DREAM estimates also showed an effect for exposure to organic solvents on clothing layer of legs whereas the original DREAM estimates did not.

\section{DISCUSSION}

For skin exposures, Spearman correlation coefficients for individual observations ranged from 0.19 to 0.82. DREAM estimates showed a statistically significant fixed effect between and within surveys for hands and forearms, explaining mainly between-task variance and to a lesser extent between individuals. In general, exposure levels on clothing layer were only predicted in a meaningful way by the detailed DREAM estimates, which comprised detailed 
Table 3 Linear mixed regression models for individual observations of skin exposure for body parts with $>50 \%$ detectable samples: logged dermal exposures $\left(\mu \mathrm{g} / \mathrm{cm}^{2}\right)$ as response variable $(Y)$, task and subject included as random effects (model $A$ ); with task and subject included as random effect and DREAM estimates as fixed effect (model B); and with task and subject included as random effect and detailed DREAM DET estimates as fixed effect (model C)

\begin{tabular}{|c|c|c|c|c|c|c|c|c|c|c|c|}
\hline \multirow[b]{2}{*}{ Agent } & \multirow[b]{2}{*}{ Body part } & \multirow[b]{2}{*}{$n^{*}$} & \multirow[b]{2}{*}{$\mathbf{K} \dagger$} & \multirow[b]{2}{*}{$\mathrm{T} \ddagger$} & \multirow[b]{2}{*}{ Model } & \multicolumn{3}{|c|}{ Random effects } & \multicolumn{2}{|c|}{ Fixed effects $\beta$ (SE) } & \multirow{2}{*}{$\begin{array}{l}\% \text { of total } \\
\text { variance } \\
\text { explained by } \\
\text { DREAM }\end{array}$} \\
\hline & & & & & & $S^{2}{ }_{\text {TASK }}$ & $\mathrm{S}^{2}{ }_{\mathrm{BW}}$ & $S^{2} w w$ & Intercept $\left(\gamma_{00}\right)$ & $\gamma_{01} \cdot \operatorname{LN}\left(D R E A M_{i}\right)$ & \\
\hline \multicolumn{12}{|l|}{ Between surveys } \\
\hline \multirow[t]{3}{*}{$C P, M W F, O S$} & Hands & 80 & 60 & 11 & A & 44.1 & 2.2 & 0.4 & $2.9(2.0)$ & & \\
\hline & & & & & $B$ & 27.4 & 1.8 & 0.3 & $3.3(1.6)$ & $0.50(0.11)^{\star \star *}$ & 37 \\
\hline & & & & & C & 15.6 & 1.8 & 0.4 & $3.9(1.2)$ & $0.57(0.10)^{\star \star \star *}$ & 62 \\
\hline \multicolumn{12}{|l|}{ Within surveys } \\
\hline \multirow[t]{4}{*}{ MWF } & Hands & 41 & 32 & 3 & $A$ & 0.7 & 2.5 & 0.6 & $6.5(0.6)$ & & \\
\hline & & & & & $B$ & 0.6 & 2.3 & 0.5 & $5.6(0.6)$ & $0.39(0.14)^{*}$ & 11 \\
\hline & Forearms & 41 & 32 & 3 & A & 0.4 & 1.4 & 1.4 & $6.4(0.5)$ & & \\
\hline & & & & & $B$ & 0.1 & 0.03 & 2.3 & $5.8(0.4)$ & $0.45(0.15)^{*}$ & 24 \\
\hline \multirow[t]{3}{*}{ OS } & Hands & 16 & 10 & 6 & A & 3.2 & 0.4 & 0.4 & $5.5(0.8)$ & & \\
\hline & & & & & $B$ & 0.7 & 0.4 & 0.2 & $5.8(0.4)$ & $0.61(0.15)^{\star *}$ & 68 \\
\hline & & & & & $\mathrm{C}$ & 1.3 & 0.4 & 0.2 & $5.8(0.5)$ & $0.60(0.18)^{*}$ & 53 \\
\hline \multirow[t]{3}{*}{ OS } & Legs & 27 & 16 & 6 & A & 2.5 & 0.05 & 0.3 & $5.7(0.7)$ & & \\
\hline & & & & & $B$ & 2.1 & 0.04 & 0.3 & $6.2(0.7)$ & $0.24(0.15)$ & 14 \\
\hline & & & & & $\mathrm{C}$ & 2.0 & 0.04 & 0.3 & $6.2(0.7)$ & $0.24(0.15)$ & 18 \\
\hline
\end{tabular}

${ }^{*}<0.05 ;{ }^{* *}<0.01 ;{ }^{* * *}<0.001 ;{ }^{* * * *}<0.0001$.

*Number of measurements, $>50 \%$ of samples should be above limit of detection.

†Number of workers.

†Number of tasks.

$\mathrm{CP}$, cyclophosphamide; MWF, metal working fluids; OS, organic solvents.

information on the concentration of the agent in the formulation to which exposure occurred.

This study had several limitations. The surveys comprised exclusively data on exposure to liquids. Future work will be necessary to study accuracy of the DREAM method for solids and vapors. In addition, variability in dermal exposure levels within surveys was limited, as only two surveys comprised measurements in more than one company and within surveys a limited number of tasks were observed. Also, for a few tasks exposure levels were measured on both skin and clothing layer, hampering a direct comparison of the performance of the DREAM method between skin and clothing layer. Furthermore, we were unable to verify whether DREAM assesses accurately the spatial variability

Table 4 Linear mixed regression models for individual observations of exposure on clothing layer for body parts with $>50 \%$ detectable samples: logged dermal exposures $\left(\mu \mathrm{g} / \mathrm{cm}^{2}\right)$ as response variable $(Y)$, task and subject included as random effects (model A); with task and subject included as random effect and DREAM estimates as fixed effect (model B); and with task and subject included as random effect and detailed DREAM DET estimates as fixed effect (model C)

\begin{tabular}{|c|c|c|c|c|c|c|c|c|c|c|c|}
\hline \multirow[b]{2}{*}{ Agent } & \multirow[b]{2}{*}{ Body part } & \multirow[b]{2}{*}{$\mathbf{n}^{*}$} & \multirow[b]{2}{*}{$\mathrm{K} \dagger$} & \multirow[b]{2}{*}{$\mathbf{T}$} & \multirow[b]{2}{*}{ Model } & \multicolumn{3}{|c|}{ Random effects } & \multicolumn{2}{|c|}{ Fixed effects $\beta$ (se) } & \multirow{2}{*}{$\begin{array}{l}\% \text { of total variance } \\
\text { explained by DREAM }\end{array}$} \\
\hline & & & & & & $\mathrm{S}_{\text {TASK }}^{2}$ & $\mathrm{~S}^{2}{ }_{\mathrm{BW}}$ & $S^{2} w w$ & Intercept $\left(\gamma_{00}\right)$ & $\gamma_{01} \cdot \operatorname{LN}\left(\right.$ DREAM $\left.M_{\mathrm{i}}\right)$ & \\
\hline \multicolumn{12}{|l|}{ Between surveys } \\
\hline \multirow[t]{3}{*}{$C P, D E G B E$, OS } & Hands & 134 & 87 & 9 & A & 52.5 & 7.3 & 0.4 & $2.5(2.4)$ & & \\
\hline & & & & & $B$ & 27.5 & 3.2 & 0.7 & $2.0(1.8)$ & $1.32(0.17)^{\star * \star *}$ & 48 \\
\hline & & & & & C & 12.4 & 1.9 & 0.9 & $3.8(1.2)$ & $1.07(0.10)^{* * * *}$ & 75 \\
\hline \multicolumn{12}{|l|}{ Within surveys } \\
\hline \multirow[t]{3}{*}{ DEGBE } & Hands & 50 & 36 & 3 & A & 18.4 & 3.3 & 0.4 & $4.3(2.7)$ & & \\
\hline & & & & & B & 19.7 & 2.9 & 0.5 & $5.0(2.7)$ & $0.68(0.25)^{*}$ & 10 \\
\hline & & & & & C & 6.7 & 1.7 & 1.4 & $6.0(1.7)$ & $0.84(0.19)^{\star * *}$ & 56 \\
\hline \multirow[t]{3}{*}{ OS } & Hands & 61 & 33 & 4 & $A$ & 1.5 & 0.1 & 0.2 & $7.0(0.6)$ & & \\
\hline & & & & & $B$ & 1.5 & 0.1 & 0.2 & $7.1(0.9)$ & $-0.03(0.23)$ & 0 \\
\hline & & & & & C & 1.3 & 0.1 & 0.2 & $6.7(0.8)$ & $0.12(0.22)$ & 13 \\
\hline \multicolumn{12}{|l|}{ Between surveys } \\
\hline \multirow[t]{3}{*}{$C P, M W F, O S$} & Legs & 124 & 82 & 10 & $A$ & 58.5 & 0.4 & 0.4 & $4.0(2.4)$ & & \\
\hline & & & & & $B$ & 47.3 & 0.2 & 0.4 & $3.9(2.2)$ & $0.22(0.05)^{\star \star \star \star}$ & 19 \\
\hline & & & & & C & 40.3 & 0.3 & 0.4 & $4.2(2.0)$ & $0.23(0.05)^{\star * * *}$ & 31 \\
\hline \multicolumn{12}{|l|}{ Within surveys } \\
\hline \multirow[t]{2}{*}{ MWF } & Legs & 56 & 44 & 4 & A & 0.3 & 0.7 & 0.4 & $9.3(0.3)$ & & \\
\hline & & & & & B & 0.01 & 0.0 & 0.9 & $8.9(0.2)$ & $0.26(0.06)^{* *}$ & 38 \\
\hline \multirow[t]{3}{*}{ OS } & Legs & 62 & 33 & 4 & A & 1.9 & 0.2 & 0.3 & $5.7(0.7)$ & & \\
\hline & & & & & $B$ & 1.9 & 0.2 & 0.3 & $4.5(1.0)$ & $0.39(0.25)$ & 0 \\
\hline & & & & & C & 0.8 & 0.2 & 0.3 & $4.2(0.8)$ & $0.55(0.22)^{*}$ & 46 \\
\hline
\end{tabular}

${ }^{*}<0.05 ;{ }^{* *}<0.01 ;{ }^{* * *}<0.001 ;{ }^{* * *}<0.0001$.

*Number of measurements, $>50 \%$ of samples should be above limit of detection.

†Number of workers.

$\ddagger$ Number of tasks.

"For this survey the mixed regression models contained the random effect variable "company" in addition to "task" and "subject". Estimated variance components for company (S2 ${ }^{2}$ COMPANY) were 10.9 (model A), 6.5 (model B), and 2.3 (model C).

$\mathrm{CP}$, cyclophosphamide; DEGBE, di-ethyl-glycol-butyl-ether; OS, organic solvents; MWF, metal working fluids. 
in dermal exposure (distribution of dermal exposure over the body surface) because few measurements of dermal exposure of other body parts than hands were available, and, if available, had large amounts of undetectable samples.

When interpreting the results of our study, one should be aware that dermal exposure measurements are not a real golden standard. The measurement methods are prone to systematic and random measurement errors, the magnitude of these errors may be distinct for the different measurement methods that were used-surrogate skin (skin exposure OS, BZ, TL), fluorescent tracer (skin exposure MWF), and removal techniques (skin exposure CP). Surrogate skin methods tend to overestimate exposure because they do not mimic removal processes like washing, and because substances tend to bind more strongly to the used receptor materials (for example, cotton and activated charcoal), than to skin. ${ }^{22}$ Fluorescent tracers strongly bind to skin molecules, neglecting losses of contaminant mass from the skin surface through evaporation processes of other processes such as washing $^{27}$ and therefore may overestimate exposure as well. Removal techniques are likely to underestimate exposure levels as the skin may have absorbed part of the substance. Also the substance may only partly be removed from the skin, as the median of reported mean wipe sample efficiencies was only $51 \% .{ }^{23}$ Fenske and colleagues compared performance of surrogate skin, hand wash and wipe methods and reported that the mean glove exposure rate $(\mathrm{mg} / \mathrm{hr})$ was 3.5 -fold higher than the wash rate, whereas the wash rate was 6.4-fold higher than the wipe rate. ${ }^{28}$

Systematic measurement error may have biased the relations of DREAM estimates with measured exposure levels between surveys; in theory the relation of DREAM estimates with real dermal exposure levels may be better or worse than the found relation. We believe, however, that the effect of systematic measurement errors was small. Even if the results of the distinct methods would differ by a factor of 10-20, this would results in a similar ranking of dermal exposure levels (fig 1).

Random error of measurement methods will bias the relations between DREAM estimates and measured dermal exposure levels between, as well as within, surveys downward, ${ }^{11}$ resulting in less correlation between measured and semiquantitative dermal exposure levels. Again, the amount of random error may differ between methods. Nevertheless, in our study on grouping of dermal exposure levels to MWF among machine operators for an epidemiological study on dermatitis, ${ }^{17}$ DREAM estimates correlated to the same extent with the results of the fluorescent tracer method VITAE $(r=0.57, \mathrm{p}<0.01)$ as with the results obtained with the surrogate skin sampling technique (pads) $(r=0.59, \mathrm{p}<0.01)$. Finally, it should be mentioned that dermal exposure measurements were expressed in mass per surface area, which might not be the most biologically relevant measure of dermal exposure, as skin uptake is not driven by the mass but by the concentration of contaminant on the skin. ${ }^{29}$

Notwithstanding the limitations described above, we were able to apply the DREAM method within six surveys comprising dermal exposure situations in a wide variety of sectors (automobile industry, hospitals, chemical industry, and shoe manufacturing) for different tasks and chemical agents. Our data showed an enormous range in dermal exposure levels (8-10 orders of magnitude) and seem to represent a significant part of the spectrum of dermal exposure levels encountered in different occupational settings in industrialised countries. In general, measured dermal exposure levels varied more than DREAM estimates did (8-10 $v$ 5-6 orders of magnitude), indicating the DREAM method results in less resolution than the measurements. Interestingly, detailed DREAM estimates varied more, and were more similar to measurements, than original DREAM estimates. The categorisation of the active ingredient of interest seems to have biased the DREAM estimates upward.

Between surveys, the DREAM method provided accurate estimates for task based as well as individual exposure levels on skin and on clothing layer of hands, explaining considerable amounts of between-task variance and, to a lesser extent, between-worker variance. The DREAM estimates did not explain variability in exposure between days. Apparently, the event based nature of dermal exposure ${ }^{30}$ is less efficiently picked up by an observational method. However, this variance component is only of interest for exposure assessment in epidemiological surveys focusing on acute effects, or effects from peak exposures.

Within surveys, for skin exposures on hands, the DREAM method was able to rank tasks for which exposure varied half, up to two orders of magnitude (two out of four surveys). The task based correlations for skin exposure of hands were based on few data points, and should be interpreted with caution. For two out of three surveys the DREAM method provided moderate estimates of individual skin exposures, explaining reasonable amounts of betweentask and between-worker variance. Within surveys, exposure levels on clothing layer of hands were only predicted in a meaningful way by the detailed DREAM estimates, which comprised detailed information on the concentration of the agent in the formulation to which exposure occurred.

Some of the DREAM estimates deviated much from the measurements and are thus interesting from a model improvement point of view. In the case of $\mathrm{CP}$, dermal exposure levels were very low. It appeared to be difficult to distinguish differences for these very low exposure levels with DREAM. However, measurement methods also encountered these problems as over $62 \%$ of the samples were below the limit of detection. For exposure to MWF, moderate correlations were found for workers who performed similar tasks-operating metal working machines. The DREAM model possibly could be improved for this specific situation by including process specific determinants such as open or closed metal working procedures, number of work parts handled by the worker in combination with the use of compressed air to clean metal parts. ${ }^{17}$ Actually, we showed elsewhere that the identified factors affecting dermal exposure were quite similar for the general DREAM method and quantitative measurement methods (VITAE, surrogate skin pads). ${ }^{17}$ With regard to OS, for skin exposures, paint spraying tasks as well as two other tasks (unmasking truck parts, mixing) were evaluated, showing good correlations between DREAM estimates and measurements. For exposure on clothing layer, only spraying tasks were included. When observing these spraying tasks, they seemed to be very similar with regard to exposure processes; dermal exposure principally resulted from deposition from paint and from transfer due to contact with contaminated surfaces. It was therefore difficult to detect differences in exposure levels with DREAM. The spraying tasks differed, however, with regard to the concentration of organic solvents, litres of paint sprayed per hour, spray pressure, and ventilation rate of the spray cabin. Inclusion of these factors in the DREAM model might improve its estimates. Inclusion of concentration in the model resulted in a significant improvement. The latter was also the case for exposure to DEGBE of the clothing layer. It is, however, important to keep in mind that a specific model for paint spraying already exists; ${ }^{7}$ the main reason for developing DREAM was to construct a generic initial assessment tool for dermal exposure assessment.

For exposures on other body parts than hands only very few data were available. For skin exposure, predictions on 
legs seemed to be less accurate than for hands (exposure to organic solvents, spray painting), whereas predictions for forearms seemed to be similar as for hands (exposure to MWF). For exposure on clothing layer, ranking of exposure levels on legs between surveys seemed to be poor when compared to ranking of hands, whereas predictions within studies seemed to be about the same as for hands.

For skin exposures within surveys, the predominantly moderate correlations of DREAM estimates with measured skin exposure levels showed that the DREAM method has difficulties predicting exposures in a meaningful way for situations with a small between-task variance $\left(\mathrm{S}^{2}{ }_{\text {TASK }}<1.0\right.$, for example, tasks in which average dermal exposure levels are within half an order of magnitude). Nonetheless, a few large scale exposure surveys on dermal exposure have shown that the variability in dermal exposure sometimes is larger than an order of magnitude. In these cases, the DREAM estimates seem specific enough to provide useful dermal exposure estimates for tasks and jobs for epidemiological surveys. An exposure survey in 94 fruit growers ${ }^{31}$ reported a between-task variance of 2.6 and 3.6 for skin exposure of hands and wrists, respectively, quite similar to the betweentask variance for skin exposure of hands of the spray painters in this study of 3.2, for which the DREAM estimates explained $68 \%$ of total variance. On the other hand, Kromhout et $a l^{32}$ and Vermeulen et $a l^{33}$ performed industry wide surveys in the rubber manufacturing industry, where variability exposure levels between production functions was less than 0.6, comparable to the variability in exposure levels between tasks of our metalworking survey (0.7) for which the DREAM estimates only explained $11 \%$ of total variance.

As far as we know, only one validation study of a generic dermal exposure method has been published in the peer reviewed literature. This study compared measured dermal dust exposures with predicted exposures given by the EASE expert system. ${ }^{34}$ EASE provides estimates for dermal exposure levels on hand and forearms and uses three dermal exposure bands: $0.1-1,1-5$, and $5-15 \mathrm{mg} / \mathrm{cm} /$ day, which are based on use patterns of the agent for which exposure is estimated. Although the average exposures increased with the predictions from EASE, the ranges of the measured exposure levels for the different categories showed quite some overlap. ${ }^{34}$ The model overestimated dermal exposure to the hands by a factor of 50 as compared with average measured exposure levels. The authors did not present correlation coefficients. A limitation of the EASE system is that exposure is only estimated for hands and forearms, disregarding dermal exposure levels of other body parts.

The performance of the DREAM method is comparable to that of a generic model for airborne exposures validated by Cherrie and Schneider $^{6}$ for five chemical agents. The correlations between logged measurements and logged estimates ranged in that study from 0.0 to 0.93 (median 0.50 ). The process-specific dermal exposure model for spray painting by Brouwer $\mathrm{et}^{\mathrm{al}} \mathrm{l}^{7}$ showed good correlation $(r=0.82)$ with measured exposure values on clothing layer (total body), performing considerably better than the DREAM method did for spray painting on clothing layer (detailed DREAM estimates $r=0.4$ (hands) and $r=0.2$ (legs). However, the validation of the dermal model for spray painting by Brouwer et $a l^{7}$ was done in a semi-experimental setting. Correlations for real life full shift exposure scenarios will probably be lower. ${ }^{13}$ Nevertheless, as already stated before, the DREAM method possibly could be improved for specific situations by adding more detailed determinants of dermal exposure to the model such as source strength in case of spray painting. The DREAM method showed comparable correlations with measured skin exposure levels on hands as compared to a study by de Cock et $a l^{4}$ who reported correlation coefficients between 0.4 and 0.7 for the ranking of dermal exposure to pesticides on wrists $(n=15)$ by five occupational hygienists.

In conclusion, the DREAM method appears to be a promising generic model for range finding of skin exposure levels, principally explaining variability in dermal exposure levels between tasks. The accuracy of the DREAM estimates within surveys varies, mainly due to differences in variability in exposure levels between tasks. For exposure on clothing layer, only detailed DREAM estimates provided meaningful exposure estimates. The DREAM method appeared to have an acceptable accuracy that may be enhanced, and calibrated for specific exposure situations. A previous study on the reliability of DREAM showed good interobserver agreement. ${ }^{16}$ We expect that the DREAM method can be successfully applied for semiquantitative dermal exposure assessment of groups of workers in epidemiological and occupational hygiene surveys with contrasting exposure levels (variability in exposure levels between groups $>1.0$ ). For surveys with less contrasting exposure levels, quantitative dermal exposure measurements are to be preferred as estimates with DREAM would only explain an insignificant part of betweengroup variability.

\section{ACKNOWLEDGEMENTS}

The authors would like to thank José Gijsbers for making data available. We are grateful to Mark Lurvink, Martijn Kerkman, Tim Meijster, and Karmen Ramakers for observing the employees, and the employers and employees for participating. We thank Dick Heederik for commenting on a previous version of this manuscript. The Dutch Ministry of Social Affairs and Employment financed this study.

\section{Authors' affiliations}

B van W de Joode, Risk Assessment in the Work Environment, a collaborative centre between TNO Quality of Life and IRAS; Currently at: Central American Institute for Studies on Toxic Substances (IRET), National University, Heredia, Costa Rica

W Fransman, H Kromhout, Environmental and Occupational Health Division, Institute for Risk Assessment Sciences (IRAS), Utrecht University, Utrecht, Netherlands

J J van Hemmen, TNO Quality of Life, Netherlands R Vermeulen, Occupational and Environmental Epidemiology Branch, Division of Cancer Epidemiology and Genetics, National Cancer Institute, Rockville, USA

\section{REFERENCES}

1 Teschke K, Olshan AF, Daniels JL, et al. Occupational exposure assessment in case-control studies: opportunities for improvement. Occup Environ Med 2002;59:575-93

2 Kromhout H, Oostendorp Y, Heederik D, et al. Agreement between qualitative exposure estimates and quantitative exposure measurements. Am J Ind Med 1987; 12:551-62.

3 Hertzman C, Teschke K, Dimich-Ward H, et al. Validity and reliability of a method for retrospective evaluation of chlorophenate exposure in the lumber industry. Am J Ind Med 1988;14:703-13.

4 de Cock J, Kromhout $\mathrm{H}$, Heederik D, et al. Experts' subjective assessment of pesticide exposure in fruit growing. Scand J Work Environ Health 1996;22:425-32

5 Segnan N, Ponti A, Ronco G, et al. Comparison of methods for assessing the probability of exposure in metal plating, shoe and leather goods manufacture and vine growing. Occup Hyg 1996;3:199-208

6 Cherrie JW, Schneider T. Validation of a new method for structured subjective assessment of past concentrations. Ann Occup Hyg 1999;43:235-45.

7 Brouwer DH, Semple S, Marquart H, et al. A dermal model for spray painters. Part I: Subjective exposure modelling of spray paint deposition. Ann Occup Hyg 2001;45:15-23.

8 Semple SE, Proud LA, Tannahill SN, et al. A training exercise in subjectively estimating inhalation exposures. Scand J Work Environ Health 2001;27:395-401.

9 Stewart PA, Lees PS, Correa A, et al. Evaluation of three retrospective exposure assessment methods. Ann Occup Hyg 2003;47:399-411.

10 Tielemans E, Heederik D, Burdorf A, et al. Assessment of occupational exposures in a general population: comparison of different methods. Occup Environ Med 1999;56:145-51. 
11 Armstrong BG. Effect of measurement error on epidemiological studies of environmental and occupational exposures. Occup Environ Med 1998;55:651-6.

12 Thomas D, Stram D, Dwyer J. Exposure measurement error: influence on exposure-disease - relationships and methods of correction. Annu Rev Public Health 1993; 14:69-93.

13 Vermeulen R, Stewart P, Kromhout H. Dermal exposure assessment in occupational epidemiologic research. Scand J Work Environ Health 2002;28:371-85.

14 Van Wendel de Joode B, Brouwer DH, Vermeulen R, et al. DREAM: a method for semi-quantitative dermal exposure assessment. Ann Occup Hyg 2003;47:71-87.

15 Schneider T, Vermeulen R, Brouwer DH, et al. Conceptual model for assessment of dermal exposure. Occup Environ Med 1999;56:765-73.

16 Van Wendel de Joode B, van Hemmen JJ, Meijster T, et al. Reliability of a semi-quantitative method for dermal exposure assessment (DREAM). J Expo Anal Environ Epidemiol 2005; 15:111-20.

17 Van Wendel de Joode B, Bierman EPB, Brouwer DH, et al. An assessment of dermal exposure to semi-synthetic metal working fluids by different methods to group workers for an epidemiological study on dermatitis. Occup Environ Med 2005 (in press)

18 Fransman W, Vermeulen R, Kromhout H. Occupational dermal exposure to cyclophosphamide in Dutch hospitals: A Pilot Study. Ann Occup Hyg 2004:48:237-44.

19 Gijsbers JH, Tielemans E, Brouwer DH, et al. Dermal exposure during filling, loading and brushing with products containing 2-(2-butoxyethoxy)ethanol. Ann Occup Hyg 2004;48:219-27.

20 Van Wendel de Joode B, Tielemans E, Vermeulen R, et al. Dermal exposure assessment to benzene and toluene using charcoal cloth pads. J Expo Anal Environ Epidemiol 2005; 15:47-50.

21 Vermeulen R, Li G, Lan Q, et al. Detailed exposure assessment for a molecular epidemiology study of benzene in two shoe factories in China. Ann Occup Hyg 2004;48:105-16
22 Soutar A, Semple S, Aitken RJ, et al. Use of patches and whole body sampling for the assessment of dermal exposure. Ann Occup Hyg 2000;44:511-18.

23 Brouwer DH, Boeniger MF, van Hemmen JJ. Hand wash and manual skin wipes. Ann Occup Hyg 2000;44:501-10.

24 Fenske RA, Leffingwell JT, Spear RC. A video imaging technique for assessing dermal exposure. I. Instrument design and testing. Am Ind Hyg Assoc J 1986;47:764-70.

25 Fenske RA, Wong SM, Leffingwell JT, et al. A video imaging technique for assessing dermal exposure. II. Fluorescent tracer testing. Am Ind Hyg Assoc J 1986;47:771-5.

26 Bierman EP, Brouwer DH, van Hemmen JJ. Implementation and evaluation of the fluorescent tracer technique in greenhouse exposure studies. Ann Occup Hyg 1998;42:467-75

27 Cherrie JW, Brouwer DH, Roff M, et al. Use of qualitative and quantitative fluorescence techniques to assess dermal exposure. Ann Occup Hyg 2000;44:519-22.

28 Fenske RA, Simcox NJ, Camp JE. Comparison of three methods for assessmentof hand exposure to Azinphos-Methyl (Guthion) during apple thinning. Appl Occup Environ Hyg 1999;14:618-23.

29 Cherrie JW, Robertson A. Biological relevant assessment of dermal exposure. Ann Occup Hyg 1995;39:387-92.

30 Kromhout $\mathrm{H}$, Vermeulen R. Temporal, personal and spatial variability in dermal exposure. Ann Occup Hyg 2001:45:257-73.

31 de Cock J, Heederik D, Kromhout $\mathrm{H}$, et al. Exposure to captan in fruit growing Am Ind Hyg Assoc J 1998;59:158-65.

32 Kromhout H, Swuste P, Boleij JS. Empirical modeling of chemical exposure in the rubber-manufacturing industry. Ann Occup Hyg 1994;38:3-22.

33 Vermeulen $\mathbf{R}$, de Hartog, Swuste $P$, et al. Trends in exposure to inhalable particulate and dermal contamination in the rubber manufacturing industry: effectiveness of control measures implemented over a nine-year period. Ann Occup Hyg 2000;44:343-54.

34 Hugson G, Cherrie JW. Comparison of measured dermal dust exposures with predicted exposures given by the EASE Expert System. Ann Occup Hyg 2005;49:111-23. 